\title{
RANDOM WALK ON COUNTABLY INFINITE ABELIAN GROUPS
}

\author{
BY
}

\author{
H. KESTEN and F. SPITZER
}

Cornell University, Ithaca, N. Y., U.S.A.

\section{Introduction}

Given a probability measure $\mu$ on a countably infinite Abelian group (SS we propose to study the properties of the potential kernels

$$
\sum_{n=0}^{\infty} \mu^{(n)}(x) \text { and } \sum_{n=0}^{\infty}\left[\mu^{(n)}(0)-\mu^{(n)}(x)\right], \quad x \in \mathbb{G} .
$$

Here 0 is the identity element of the (additive) group $\left(\mathcal{G}, \mu^{(0)}\right.$ is the probability measure all of whose mass is concentrated at $0, \mu^{(1)}=\mu$ and $\mu^{(n)}$ is the $n$-fold convolution of $\mu$ with itself.

Roughly speaking, the purpose of this paper is to imitate and extend basic results in [10] (Chapter 7 and parts of earlier chapters). There the attention was strictly confined to the groups $\mathbb{G}=Z_{d}$, the groups of $d$-dimensional integers, or lattice points in Euclidean space of dimension $d$. Thus the basic ideas, methods, and notation are exactly those in [10] when possible-and most of the difficulties which arise because (S) is more complicated than $Z_{d}$ can be overcome by the use of certain measures induced by the given measure $\mu$ on cyclic subgroups of $\mathbb{S}$.

It will be assumed throughout that the measure $\mu$ is aperiodic, i.e. that the support of $\mu$ generates all of $(B)$. (Note however that $(\mathcal{S}$ must be infinite. When (G) is finite everything we do is either trivial or well known but the results are by no means the same.) Given $\mu$ we define on (S) the Markov process (random walk) $X_{n}$ with transition function

$$
\begin{aligned}
& P_{x}\left[X_{1}=y\right]=P(x, y)=\mu(y-x), \\
& P_{x}\left[X_{n}=y\right]=P_{n}(x, y)=\mu^{(n)}(y-x), \quad x, y \in \text { SS, } n \geqslant 0 .
\end{aligned}
$$

Here $P_{x}[\cdot]$ is the probability measure induced by the joint probabilities for finite paths starting at $X_{0}=x$, and the associated expectation will be denoted by $E_{x}[\cdot]$. 
We shall have occasion to study real valued functions on $(\mathcal{G}$, such as the potential kernels defined below. In studying their asymptotic behavior it will be convenient to write

$$
\lim _{|x| \rightarrow \infty} f(x)=L \text { or } \lim _{|x| \rightarrow \infty} f(x)=+\infty
$$

with the following interpretation: take an arbitrary one to one mapping $\varphi$ : $(\mathfrak{S} \rightarrow$ positive integers, and let

$$
\lim _{|x| \rightarrow \infty} f(x)=\lim _{n \rightarrow \infty} f\left[\varphi^{-1} \lambda(n)\right]
$$

This limit is independent of $\varphi$. Similarly, given a sequence $\left\{x_{n}\right\} \subset \mathbb{S}$, we shall write $\left|x_{n}\right| \rightarrow \infty$ when the sequence of integers $\varphi\left(x_{n}\right)$ tends to infinity with $n$.

We shall call the random walk (or the measure $\mu$ ) transient if

$$
G(x, y)=\sum_{n=0}^{\infty} P_{n}(x, y)=\sum_{n=0}^{\infty} \mu^{(n)}(y-x)<\infty, \quad x, y \in(B)
$$

and recurrent (or persistent) otherwise. It is well known that in the recurrent case the series defining the kernel $G(x, y)$ diverges for all $x, y$. It is then that the second kernel in (1.1) becomes an object of interest-to prove ist existence is actually our primary goal, and when it does exist it will be denoted by

$$
A(x, y)=\sum_{n=0}^{\infty}\left[P_{n}(0,0)-P_{n}(x, y)\right], \quad x, y \in \mathbb{G}
$$

Clearly $A(x, y)=A(x-y, 0)$ and $G(x, y)=G(x-y, 0)$. We therefore also use the notation

$$
a(x)=A(x, 0), \quad g(x)=G(x, 0), \quad x \in \mathcal{G}
$$

Finally, to give the probability interpretation of the distinction between transient and recurrent random walks,

$$
P_{x}\left[X_{n}=y \text { for infinitely many values of } n\right]=1
$$

if $\mu$ is recurrent and 0 if it is transient. The usual proof due to Chung and Fuchs [4] for $\mathfrak{G}=Z_{d}$ (and also for the continuous group $R_{d}$ ) requires no modification.

There is another, more recent ([10], p. 85) form of the classification of $\mu$ into the recurrent and transient type. It states that $\mu$ is transient if and only if the real part of $[1-\hat{\mu}]^{-1}$ is integrable on $\Gamma$, where $\hat{\mu}$ is the Fourier transform of $\mu$ and $\Gamma$ the (compact) character group of $(\mathcal{G}$. This theorem is proved in the next section (\$2) because it yields to somewhat easier methods than those in $\S 3$ and 4 . Moreover, it 
furnishes a striking example of our inability to extend the theory to locally compact Abelian groups. The following conjecture is still open even in the case when $\mathfrak{G}=R$, the group of real numbers:

Conjecture: Let $\mathbb{S S}$ be an infinite locally compact Abelian group, $\mu$ a probability measure on $(\mathfrak{s}$ whose support is not contained in a closed subgroup, $\hat{\mu}$ the Fourier transform of $\mu$ and $\Gamma$ the character group of $B 5$. Then

$$
\sum_{n=0}^{\infty} \mu^{(n)}(U)<\infty
$$

for some neighborhood $U$ of the origin in $(S)$ if and only if

$$
\int_{V} \operatorname{Re} \frac{1}{1-\hat{\mu}(\lambda)} d \lambda<\infty
$$

for some neighborhood $V$ of the origin in $\Gamma$, with $d \lambda$ Haar measure on $\Gamma$.

In $\S 3$ we establish the existence of the potential kernel $a(x)=A(x, 0), x \in \mathscr{G}$, for arbitrary $\mu$ and arbitrary (S) (Theorem 3.2). Just as in [10] where $\mathbb{B}=Z_{d}$, this theorem is closely related to the asymptotic behavior of hitting probabilities of finite sets (treated in Theorem 3.1), and to the asymptotic behavior of $a(x+y)-a(x)$ as $|x| \rightarrow \infty$. It is shown (in Theorem 3.3) that $a(x+y)-a(x)$ tends to zero as $|x| \rightarrow \infty$ unless (G) has an infinite cyclic subgroup $\mathfrak{S}$ such that $\mathfrak{S} / \mathfrak{S}$ is finite. And even then the limit is zero unless the random walk, observed only when it visits $\mathfrak{S}$, has mean zero and finite variance. Conversely, if $\mathfrak{S}$ is an infinite cyclic subgroup of $\mathfrak{G}$ and if the random walk, observed only when it is in $\mathfrak{H}$, has mean 0 and finite variance, then (S) $/ \mathfrak{S}$ must be a finite group (Lemma 3.4). In this case we obtain results analogous to $\mathrm{T} 29.1$ in [10] where it is shown that for random walk on $Z_{1}$, with mean zero and variance $\sigma^{2}<\infty, a(x+y)-a(x)$ tends to $\pm y / \sigma^{2}$ as $x \rightarrow \pm \infty$.

In $\S 4$ we first establish properties of the potential kernels $A(x, y)$ and $G(x, y)$, which are valid for every measure $\mu$, and which are independent of the structure of the group (5). These properties (Theorem 4.1 (a)-4.1 (b)) may be regarded as a general form of the renewal theorem, as they concern the asymptotic behavior of $a(x)$ and $g(x)$. In the special case when $\mathfrak{G}=Z_{1}$ Theorem 4.1 contains one new result, to the effect that $a(x)$ has limits as $x \rightarrow+\infty$ and as $x \rightarrow-\infty$ (which need not both be infinite!). In Theorem 4.2 the asymptotic behavior of $g(x)$ and of $a(x)$ is related to the algebraic structure of $(\mathcal{S}$ by showing that $g(x)$ and $a(x)$ can fail to tend to zero, respectively $\infty$, as $|x| \rightarrow \infty$, only when $\mathfrak{G}$ has an infinite cyclic subgroup $\mathfrak{H}$ such that $(\mathfrak{S} / \mathfrak{S}$ is finite. Theorems 4.3 and 4.4 are devoted to transient random walk on groups 16-652933 Acta mathematica 114. Imprimé le 15 octobre 1965. 
of the above type, giving necessary and sufficient conditions for $g(x)$ to have a non zero limit. The analogous problem for recurrent random walk is open even when $\mathbb{B}=Z_{1}$. Finally, Theorem 4.5 , combined with Theorem 3.3 yields a complete description of the possible boundaries of a recurrent random walk on $\mathbb{G}$, as defined by Kemeny and Snell [8]. These results are just what might have been conjectured in view of the known facts $([10], \S 31)$ ) when $\left(\mathfrak{S}=Z_{d}\right.$.

\section{The classification of random walk}

Given a probability measure $\mu$ on a countably infinite Abelian group $\mathfrak{G}$, such that the support of $\mu$ generates $(\mathfrak{S}$, we let

$$
\hat{\mu}(\lambda)=\sum_{\Theta}(x, \lambda) \mu(x) . \quad \lambda \in \Gamma,
$$

denote the Fourier transform of $\mu$, in the notation of Rudin [9].

THEOREM 2.1 .

$$
\sum_{n=0}^{\infty} \mu^{(n)}(0)<\infty \text { if and only if } \int_{\Gamma} \operatorname{Re} \frac{1}{1-\hat{\mu}(\lambda)} d \lambda<\infty .
$$

Proof. The Haar measure $d \lambda$ on $\Gamma$ is normalized so that the Fourier inversion theorem holds. Therefore

For $0 \leqslant t<1$,

$$
\mu^{(n)}(x)=\int_{\Gamma} \overline{(x, \lambda)}[\hat{\mu}(\lambda)]^{n} d \lambda, \quad x \in \mathbb{S} .
$$

$$
\sum_{n=0}^{\infty} t^{n} \mu^{(n)}(0)=\int_{\Gamma} \frac{1}{1-t \hat{\mu}(\lambda)} d \lambda=\int_{\Gamma} \operatorname{Re} \frac{1}{1-t \hat{\mu}(\lambda)} d \lambda
$$

Letting $t \nearrow 1$, and using the lemma of Fatou,

$$
\sum_{n=0}^{\infty} \mu^{(n)}(0)=\lim _{t \rightarrow 1} \int_{\Gamma} \operatorname{Re} \frac{1}{1-t \hat{\mu}(\lambda)} d \lambda \geqslant \int_{\Gamma} \lim _{t \gg 1} \operatorname{Re} \frac{1}{1-t \hat{\mu}(\lambda)} d \lambda=\int_{\Gamma} \operatorname{Re} \frac{1}{1-\hat{\mu}(\lambda)} d \lambda .
$$

The applicability of Fatou's lemma above rested on the fact that

$$
\operatorname{Re} \frac{1}{1-t \hat{\mu}}=\frac{1-t \operatorname{Re} \hat{\mu}}{|1-t \hat{\mu}|^{2}} \geqslant 0 .
$$

Therefore we have proved the easy part of Theorem 2.1, to the effect that the integral over $\Gamma$ of $\operatorname{Re}[1-\hat{\mu}]^{-1}$ is finite when $\mu$ is transient. 
To go the other way we shall now assume that $\mu$ is recurrent, so that

We define the integrals

$$
\sum_{n=0}^{\infty} \mu^{(n)}(0)=\infty
$$

and the sums

$$
I(x)=2 \int_{\Gamma}[1-\operatorname{Re}(x, \lambda)] \operatorname{Re} \frac{1}{1-\hat{\mu}(\lambda)} d \lambda, \quad x \in(S)
$$

$$
S(x)=\sum_{n=0}^{\infty}\left[2 \mu^{(n)}(0)-\mu^{(n)}(x)-\mu^{(n)}(-x)\right], \quad x \in \mathfrak{G},
$$

and shall proceed to prove that

(i) the integrand in $I(x)$ is integrable on $\Gamma$ for every $x \in(G)$;

(ii) the series $S(x)$ converges (not necessarily absolutely) for each $x \in \mathcal{S}$, and its sum is $I(x)$;

(iii) $S(x)$ also possesses the representation

$$
S(x)=E_{x}\left[N_{x}\right], \text { for } x \in \mathfrak{S}, \quad x \neq 0,
$$

where $N_{x}$ is the number of visits of the process $X_{n}$ to the point $x$ before the first visit to zero (counting the visit at time 0 ).

It is easy to see that (i) (ii), (iii) will complete the proof of Theorem 2.1. For if $\operatorname{Re}[1-\hat{\mu}(\lambda)]^{-1}$ were integrable on $\Gamma$ then we would have from (i)

$$
I(x) \leqslant 4 \int_{\Gamma} \operatorname{Re} \frac{1}{1-\hat{\mu}(\lambda)} d \lambda<\infty
$$

On the other hand, $I(x)=S(x)$ according to (ii) and the probabilistic representation in (iii) yields

$$
\lim _{|x| \rightarrow \infty} S(x)=\sum_{n=0}^{\infty} \mu^{(n)}(0)=+\infty
$$

(This last fact depends on an easy calculation, given in [10], page 86. Note however that the infinite order of the group $(\mathfrak{S}$ is here used in an essential way. However, when (S) is finite Theorem 2.1 is obviously true.)

Proof of (i). Since $\mu$ is recurrent we can select, for every $x \in(\mathfrak{G}$, a positive integer $n=n(x)$ such that $\mu^{(n)}(x)=c>0$. For this particular value of $n$,

$$
1-\operatorname{Re}(\hat{\mu}(\lambda))^{n}=\sum_{y \in(S)}[1-\operatorname{Re}(y, \lambda)] \mu^{(n)}(y) \geqslant c[1-\operatorname{Re}(x, \lambda)],
$$


and therefore

$$
\left|\frac{1-\operatorname{Re}(x, \lambda)}{1-\hat{\mu}(\lambda)}\right|=\frac{1-\operatorname{Re}(x, \lambda)}{\left|1-[\hat{\mu}(\lambda)]^{n}\right|}\left|1+\hat{\mu}+\ldots+\hat{\mu}^{n-1}\right| \leqslant n \frac{1-\operatorname{Re}(x, \lambda)}{1-\operatorname{Re}(\hat{\mu})^{n}} \leqslant \frac{n}{c}<\infty .
$$

Therefore the integrand defining $I(x)$ is integrable, which proves (i).

Proof of (ii). Using the Fourier inversion theorem, the partial sums of $S(x)$ are

$$
\sum_{k=0}^{n}\left[2 \mu^{(k)}(0)-\mu^{(k)}(x)-\mu^{(k)}(-x)\right]=2 \int_{\Gamma} \frac{1-\operatorname{Re}(x, \lambda)}{1-\hat{\mu}(\lambda)}\left[1-(\hat{\mu}(\lambda))^{n+1}\right] d \lambda .
$$

We saw in the proof of (i) that $|[1-\operatorname{Re}(x, \lambda)] /[1-\hat{\mu}(\lambda)]|$ is a bounded function on $\Gamma$ for each $x \in \mathbb{G}$. Therefore the dominated convergence theorem will complete the proof of (ii) provided that the sequence $1-\hat{\mu}^{n+1}$ converges to one almost everywhere on $\Gamma$. We shall now show that indeed $1-\hat{\mu}^{n+1}$ converges to one except possibly at a finite number of points. We let $m$ denote the greatest common divisor of the positive integers $k$ such that $\mu^{(k)}(0)>0$, and consider the measure $\nu=\mu^{(m)}$. It will clearly suffice to prove that $\hat{\nu}^{n}(\lambda) \rightarrow 0$ except at finitely many points of $\Gamma$. We call $\mathfrak{S}$ the subgroup of $\mathbb{S S}$ generated by the support of $v$, and $\Lambda=[\lambda \mid \lambda \in \Gamma, \hat{\nu}(\lambda)=1]$ the so called annihilator of $\mathfrak{g}$. Observe that $\Lambda$ is exactly that subset of $\Gamma$ where $\hat{\nu}^{n}$ does not tend to zero as $n \rightarrow \infty$. (This last set consists of the points where $|\hat{\nu}(\lambda)|=1$ but because $v^{(n)}(0)>0$ for all sufficiently large $n$ we know that $|\hat{v}(\lambda)|=1$ if and only if $\hat{v}(\lambda)=1$.) Thus it only remains to prove that $\Lambda$ is a finite set, and for this purpose we invoke the Pontryagin duality theorem in the form (Rudin [9], p. 35) which asserts that $\Lambda$ is homeomorphically isomorphic to the character group of $\mathfrak{B S} / \mathfrak{h}$. Thus $\Lambda$ is finite if and only if $\mathfrak{S} / \mathfrak{S}$ is finite. But $\mathfrak{S S} / \mathfrak{S}$ is finite, (in fact it is a group of order $m$ ) since (G) decomposes into the cosets $\mathfrak{S}+g_{k}, k=0, \ldots, m-1$, where $\mathfrak{T}+g_{k}$ is the set of points $x$ of $\mathbb{G}$ such that $\mu^{n}(x)>0$ only when $n \equiv k(\bmod m)$. (For a detailed proof of this assertion, see [10], p. 43.)

Proof of (iii). We take an arbitrary point $b \neq 0$ in (S) and proceed to develop the elementary potential theory associated with the hitting probabilities of the two point set $B=\{0, b\}$. Actually the relevant parts of the theory of random walk on $\mathbb{G}=Z_{2}$ in $[10]$, Chapter 3 , apply to an arbitrary countable group $\mathbb{B}$ without modification, and so it will suffice to outline the theory. One defines

$H_{B}(x, y)=P_{x}[$ the first visit to $B$ occurs at the point $y$ ] when $x \in \mathbb{S}-B, y \in B$,

$H_{B}(x, y)=\delta(x, y)=1$ for $x=y, 0$ for $x \neq y$, when $x, y \in B$. 
when $x, y \in B$, just as in definition D 10.1 of [10]. It is then easily shown (as in $\mathrm{P}$ 11.3 of [10]) that for all $x \in \mathbb{S}$

$$
\begin{aligned}
\sum_{t \in(B)} P_{n+1}(x, t) H_{B}(t, 0) & =H_{B}(x, 0)-\sum_{t \in B} a_{n}(x-t)\left[\left[\prod_{B}(t, 0)-\delta(t, 0)\right]\right. \\
& =H_{B}(x, 0)+\left[a_{n}(x)-a_{n}(x-b)\right] \prod_{B}(b, 0)
\end{aligned}
$$

where

$$
a_{n}(x)=\sum_{k=0}^{n}\left[\mu^{(k)}(0)-\mu^{(k)}(-x)\right]
$$

Specializing to $x=b$ and $x=0$ in equation (2.3),

$$
\begin{gathered}
\sum_{t \in \mathbb{G}} P_{n+1}(b, t) H_{B}(t, 0)=a_{n}(b) \prod_{B}(b, 0), \\
\sum_{t \in \mathbb{S}} P_{n+1}(0, t) H_{B}(t, 0)=-a_{n}(-b) \prod_{B}(b, 0)+1
\end{gathered}
$$

At this point it is important to know that every bounded non-negative solution $f$ of

$$
P f(x)=\sum_{y \in \mathbb{S}} P(x, y) f(y)=\sum_{y \in \mathscr{S}} \mu(y-x) f(y)=f(x), \quad x \in \mathbb{S},
$$

is constant on $G$. This was proved by Choquet and Deny [2] (see also P 13.1 in [10] where it is shown under the weaker hypothesis that $f \geqslant 0$, when $\mu$ is recurrent). Letting $n^{\prime}$ denote a subsequence of the integers such that

$$
\lim _{n^{\prime} \rightarrow \infty} \sum_{t \in \mathscr{G}} P_{n^{\prime}+1}(x, t) H_{B}(t, 0)=\phi(x)
$$

exists for all $x \in \mathbb{S}$ it is easily seen that the limit satisfies $P \phi(x)=\phi(x), x \in \mathbb{S}$. (One has to observe that

$$
\phi_{n}(x)=\sum_{t \in G} P_{n+1}(x, t) H_{B}(t, 0)
$$

satisfies $\phi_{n+1}(x)-\phi_{n}(x) \rightarrow 0$ as $n \rightarrow \infty$.) This follows from equation (2.5) using the fact that

$$
a_{n+1}(x)-a_{n}(x)=\mu^{(n+1)}(0)-\mu^{(n+1)}(-x)=o(1), x \in \mathbb{S},
$$

as $n \rightarrow \infty$. (The simplest proof of (2.9) depends on the theory of Markov chains. The invariant measure of the random walk, being group-invariant, is constant and hence of total infinite mass. Since all states communicate, the chain is irreducible, and it 
follows (see [6], page 356) that all $n$-step transition probabilities tend to zero as $n \rightarrow \infty$.)

The final step of the proof depends on equations (2.5) and (2.6). Setting $n=n^{\prime}$, subtracting (2.6) from (2.5), and letting $n^{\prime} \rightarrow \infty$, yields

$$
1=\lim _{n^{\prime} \rightarrow \infty}\left[a_{n^{\prime}}(b)+a_{n^{\prime}}(-b)\right] \prod_{B}(b, 0) .
$$

Referring to the definition of $a_{n}(x)$ and to the series defining $S(x)$ in (ii) at the beginning of this section, we have proved

$$
1=S(b) \Pi_{B}(b, 0)
$$

The definition of $\Pi_{B}$ in (2.2) implies that $\left[\Pi_{B}(b, 0)\right]^{-1}$ is the expected number of visits of the random walk, starting at $b$, to the point $b$ before the first visit to 0 (counting the visit to $b$ at time 0 ). Since $b$ is an arbitrary point other than 0 , that completes the proof of (iii) and hence of Theorem 2.1.

\section{The potential kernel $A(x, y)$}

In view of equation (2.3) it is possible to reduce the proof of existence of the potential kernel $A(x, y)$ to the study of the limit

$$
\varphi(x)=\lim _{n \rightarrow \infty} \sum_{t \in \mathcal{G}} P_{n+1}(x, t) H_{B}(t, 0) .
$$

Here, and in the sequel, $B$ is the set $\{0, b\}$, where $b$ is fixed, and non-zero. If the limit in (3.1) exists, then as pointed out in connection with equation (2.8), it will be independent of $x$, and the existence of $A(b, 0)$ will follow readily from equation (2.3) or (2.5). In order to prove the existence of the limit in (3.1) we shall make use of known facts from [10] concerning one dimensional random walk, by studying the imbedded random walk on the cyclic group $\mathfrak{H}$ which is generated by the fixed element $b$.

Let $0=z_{0}, z_{1}, z_{2}, \ldots$ denote a fixed set of representatives for the cosets of $\mathfrak{W}$. Then each element of $\mathbb{S S}$ may be represented uniquely in the form $z_{i}+k b$, and $\mathbb{S}$ itself has the form $\mathfrak{S}=\bigcup \mathbb{U}\left\{z_{i}+\mathfrak{g}\right\}$. To define the imbedded random walk let the random variables $0<T_{1}<T_{2}<\ldots$ denote the times of successive visits of $X_{n}$ to $\mathfrak{S}$, i.e.,

$$
X_{T_{i}} \in \mathfrak{S} \text { but } X_{j} \notin \mathfrak{L} \text { when } j \geqslant 1 \text { and } j \neq T_{i} \text { for all } i \geqslant 1 \text {. }
$$

We define

$$
U_{i}=X_{T_{i}}-T_{T_{i-1}} \text { for } i \geqslant 2, U_{1}=X_{T_{1}}-X_{0},
$$

so that the random variables $U_{i}$ are independent and moreover identically distributed 
(with the exception of $U_{1}$ which has the same distribution as $U_{i}, i \geqslant 2$, only if $X_{0} \in \mathfrak{S}$ ). $X_{T_{k}}=U_{1}+\ldots+U_{k}$ is then the imbedded random walk. We may identify it with a random walk on the integers by mapping $U_{i}$ into the integer $k=k\left(U_{i}\right)$ when $U_{i}=k b$. This enables us to talk about moments of the imbedded random walk, in particular its variance

$$
\sigma^{2}=\sigma^{2}\left[k\left(U_{2}\right)\right] \leqslant \infty
$$

Henceforth, to simplify notation we shall not distinguish between $U_{i}$ and $k\left(U_{i}\right)$ and only talk about $U_{i}$.

The variance $\sigma^{2}$ is the basis for the following classification:

I $\mathfrak{H}$ is finite,

II $\mathfrak{S}$ is infinite and $\sigma^{2}=\infty$,

III $\mathfrak{H}$ is infinite and $\sigma^{2}<\infty$.

Just as $H_{B}(x, y)$ denotes the hitting probabilities for $X_{n}$ (see equation (2.1) or D 10.1 in [10]) we use $\bar{H}_{B}(x, y), x, y \in \mathfrak{H}$, to denote the hitting probabilities for the imbedded random walk. We know already (T 30.1 in [10]) that

$$
\lim _{|k| \rightarrow \infty} \bar{H}_{B}(k b, 0) \text { exists in case } I I
$$

and the two limits $\quad \lim _{k \rightarrow \pm \infty} \bar{H}_{B}(k b, 0)$ exist in case III,

and we proceed to connect these limits to $\varphi$ in equation (3.1). The precise relation is given in the following theorem.

THEOREM 3.1. In case I,

$$
\lim _{n \rightarrow \infty} \sum_{t \in \mathscr{G}} P_{n+1}(x, t) H_{B}(t, 0)=\lim _{|x| \rightarrow \infty} H_{B}(x, 0)=\frac{1}{h} \sum_{k=0}^{h-1} \bar{H}_{B}(k b, 0),
$$

where $h$ is the order of $b$.

In case II,

$$
\lim _{n \rightarrow \infty} \sum_{t \in \Theta} P_{n+1}(x, t) H_{B}(t, 0)=\lim _{|x| \rightarrow \infty} H_{B}(x, 0)=\lim _{|k| \rightarrow \infty} \bar{H}_{B}(k b, 0) .
$$

In case $\mathrm{IIT}, \mathfrak{G H} / \mathfrak{S}$ is finite and for each $i$

$$
\begin{aligned}
\lim _{n \rightarrow \infty} \sum_{t \in \mathscr{G}} P_{n+1}(x, t) H_{B}(t, 0) & =\frac{1}{2} \lim _{k \rightarrow \infty}\left\{H_{B}\left(z_{i}+k b, 0\right)+H_{B}\left(z_{i}-k b, 0\right)\right\} \\
& =\frac{1}{2} \lim _{k \rightarrow \infty}\left\{\bar{H}_{B}(k b, 0)+\bar{H}_{B}(-k b, 0)\right\},
\end{aligned}
$$


In the first two cases the proof is based on the relation

$$
H_{B}(x, 0)=\sum_{k b \in \varsubsetneqq} P_{x}\left[X_{T_{1}}=k b\right] \bar{H}_{B}(k b, 0)
$$

which follows immediately from the interpretation of $H_{B}$ and $\bar{H}_{B}$ as hitting probabilities. In case $I$, the second equality in (3.4) will follow from (3.7) and

$$
\lim _{|x| \rightarrow \infty} P_{x}\left[X_{T_{1}}=k b\right]=\frac{1}{h}, \quad k=0,1, \ldots, h-1
$$

In case II, it will suffice for the second equality in (3.5) to show

$$
\lim _{|x| \rightarrow \infty} P_{x}\left[X_{T_{1}}=k b \quad \text { with }|k| \leqslant l\right]=0
$$

for each fixed $l$, because of $(3.2),(3.7)$ and

$$
\sum_{k=-\infty}^{+\infty} P_{x}\left[X_{T_{1}}=k b\right]=1
$$

In fact, these relations show that

$$
\begin{aligned}
\left|H_{B}(x, 0)-\lim _{|k| \rightarrow \infty} \bar{H}_{B}(k b, 0)\right| \leqslant & P_{x}\left[X_{T_{1}}=k b \text { with }|k| \leqslant l\right] \\
& +\sum_{|k|>l} P_{x}\left[X_{T_{1}}=k b\right]\left|\bar{H}_{B}(k b, 0)-\lim _{m \rightarrow \infty} \bar{H}_{B}(m b, 0)\right| \\
& =o(1) \text { as first }|x| \rightarrow \infty \text { and then } l \rightarrow \infty .
\end{aligned}
$$

In case III a new argument is needed to show that $\mathfrak{G} / \mathfrak{S}$ is finite. The second equality in (3.6) will then be very easy. The first equalities in (3.4) and (3.5) will follow quite easily from (3.8) and (3.9) but in case III we need again a special argument for (3.6).

We now prove (3.8), (3.9) and the finiteness of $(\mathfrak{S} / \mathfrak{S}$ in case III in a number of lemmas.

LЕммА 3.1. For each fixed $y \in \mathbb{S}$ and $k$

$$
\lim _{i \rightarrow \infty} \sup _{x \in\left\{z_{i}+\mathfrak{b}\right\}}\left|P_{x}\left[X_{T_{1}}=k b\right]-P_{x+y}\left[X_{T_{1}}=k b\right]\right|=0 \text {. }
$$

Proof.

$P_{x}\left[X_{T_{1}}=k b\right]=P_{x}$ [enter $\mathfrak{S}$ for the first time at $\left.k b\right]$

$$
\left.\geqslant P_{x} \text { [visit } x+y \text { before visiting } \mathfrak{S}\right] P_{x+y}\left[X_{T_{\mathbf{l}}}=k b\right] \text {. }
$$


However, if $-x \in\left\{z_{j(x)}+\mathfrak{S}\right\}$, then

$P_{x}[$ visit $x+y$ before visiting $\mathfrak{S}]=P_{0}\left[\right.$ visit $y$ before visiting $\left.\left\{z_{j(x)}+\mathfrak{S}\right\}\right]$

and by the recurrence of the random walk

$$
\lim _{j \rightarrow \infty} P_{0}\left[\text { visit } y \text { before visiting }\left\{z_{j}+\mathfrak{S}\right\}\right]=1 \text {. }
$$

Since $x \in\left\{z_{i(x)}+\mathfrak{h}\right\},-x \in\left\{z_{j(x)}+\mathfrak{H}_{\mathcal{B}}\right\}$ and $i(x) \rightarrow \infty$ imply $j(x) \rightarrow \infty$, we conclude

$$
\liminf _{i \rightarrow \infty} \inf _{x \in\left\{z_{i}+\mathfrak{g}\right\}}\left\{P_{x}\left[X_{T_{1}}=k b\right]-P_{x+y}\left[X_{T_{1}}=k b\right]\right\} \geqslant 0 .
$$

Replacing $x$ by $x+y$ and $y$ by $-y$ we also have

$$
\begin{aligned}
& \liminf _{i \rightarrow \infty} \inf _{x \in\left\{z_{i}+\xi\right\}}\left\{P_{x+y}\left[X_{T_{1}}=k b\right]-P_{(x+y)-y}\left[X_{T_{1}}=k b\right]\right\}
\end{aligned}
$$

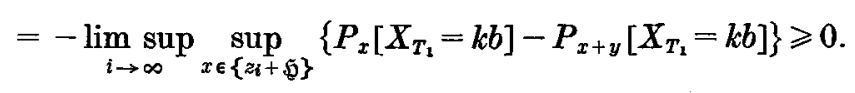

(3.12) and (3.13) prove the lemma.

LFMмA 3.2. (3.8) holds in case I.

Proof. Since

$$
P_{x+m b}\left[X_{T_{1}}=k b\right]=P_{x}\left[X_{T_{1}}=(k-m) b\right]
$$

we have

$$
\sum_{m=0}^{n-1} P_{x+m b}\left[X_{T_{1}}=k b\right]=\sum_{m=0}^{n-1} P_{x}\left[X_{T_{1}}=(k-m) b\right]=P_{x}\left[X_{T_{1}} \in \mathfrak{g}\right]=1
$$

If $x \in\left\{z_{\mathfrak{i}(x)}+\mathfrak{S}\right\}$ then $|x| \rightarrow \infty$ is equivalent to $i(x) \rightarrow \infty$ for finite $\mathfrak{S}$. Therefore (3.8) follows from (3.15) and (3.10) in case $I$.

LEMMA 3.3. (3.9) holds in case II.

Proof. Let $x=z_{i(x)}+m(x) b$. From every sequence $\{x\}$ of elements in $(G)$ for which $|x| \rightarrow \infty$ we can select a subsequence for which either

$$
\text { (i) }|m(x)| \rightarrow \infty, i(x)=i=\text { constant } \quad \text { or } \quad \text { (ii) } i(x) \rightarrow \infty \text {. }
$$

For sequences satisfying (i) we have by (3.14)

$$
\lim _{|m| \rightarrow \infty} P_{z_{i}+m b}\left[X_{T_{1}}=k b\right]=\lim _{|m| \rightarrow \infty} P_{z_{i}}\left[X_{T_{1}}=(k-m) b\right]=0
$$


for each fixed $k$, and thus (3.9). On the other hand, for a sequence satisfying (ii) we have by Lemma 3.1 and (3.14)

$$
\begin{aligned}
\limsup _{i \rightarrow \infty} \sup _{m} P_{z_{i}+m b}\left[X_{T_{1}}=k b\right] & =\lim _{i \rightarrow \infty} \sup \frac{1}{n+1} \sup _{m} \sum_{j=m}^{m+n} P_{z_{i}+m b}\left[X_{T_{1}}=k b\right] \\
& =\frac{1}{n+1} \lim \sup \sup _{i \rightarrow \infty} \sum_{j=m}^{m+n} P_{z_{i}}\left[X_{T_{1}}=(k-m) b\right] \leqslant \frac{1}{n+1} .
\end{aligned}
$$

This holds for each $n$ and therefore (3.9) holds no matter how $|x| \rightarrow \infty$.

In order to deal with case III we first show that it can only occur if $\mathfrak{G H} / \mathfrak{S}$ is finite.

LEMMA 3.4. $\mathfrak{S}$ infinite and $\sigma^{2}<\infty$ implies that $\mathbb{G S} / \mathfrak{S}$ is finite.

Proof. We give an indirect proof. We assume therefore that both $(S)$ and $\mathbb{G S} / \mathfrak{S}$ are infinite and derive from these assumptions that $\sigma^{2}=\infty$. To avoid some minor technical difficulties we give the proof only under the additional assumption:

there exist $r_{1}, r_{2}$ such that $r_{1} \neq r_{2}$ and $p_{1}=P\left(0, r_{1} b\right)>0$ and $p_{2}=P\left(0, r_{2} b\right)>0$.

Let then $\quad N=$ number of $j \leqslant T_{1}$ for which $X_{j}-X_{j-1}=r_{1} b$ or $r_{2} b$.

By the exponential estimates for the tails of a binomial distribution ([10], p. 45) one has

$$
\begin{aligned}
P_{0}\left[T_{1}=t\right. & \text { and } \left.\left|N-\left(p_{1}+p_{2}\right) t\right| \geqslant \varepsilon t\right] \\
& \leqslant P_{0}\left[1 / t \mid\left(\text { number of } j \leqslant t \text { with } X_{j}-X_{j-1}=r_{1} b \text { or } r_{2} b\right)-\left(p_{1}+p_{2}\right) \mid \geqslant \varepsilon\right] \\
& \leqslant C e^{-\lambda t}
\end{aligned}
$$

for suitable $C=C(\varepsilon)$ and $\lambda=\lambda(\varepsilon)$. Let $T$ and $N$ be fixed, say $t, n$, and assume that also the indices $1 \leqslant j_{1}<j_{2}<\ldots<j_{n} \leqslant t$ for which $X_{j}-X_{j-1}$ equals $r_{1} b$ or $r_{2} b$ are fixed as well as the values of $X_{k}-X_{k-1}$ for $k \notin\left\{j_{1}, j_{2}, \ldots, j_{n}\right\}$. We claim that, conditional upon $T=t, N=n, X_{j}-X_{j-1}=r_{1} b$ or $r_{2} b$ only for $j \in\left\{j_{1}, \ldots, j_{n}\right\}$ and finally,

$$
X_{k}-X_{k-1}=g_{k}\left(\neq r_{1} b \text { or } r_{2} b\right) \text { for } k \notin\left\{j_{1}, \ldots, j_{n}\right\} \text {, }
$$

the random variables $X_{j_{i}}-X_{j_{i-1}}, i=1, \ldots, n$ are independent and each have the same distribution

$$
P\left[X_{f_{i}}-X_{j_{i-1}}=r_{l} b\right]=\frac{p_{l}}{p_{1}+p_{2}}, \quad l=1,2
$$

Indeed, this follows immediately from the fact that $X_{1}-X_{0}, X_{2}-X_{1}, \ldots$ are inde- 
pendent random variables and the fact that changing $X_{j}-X_{j-1}$ from $r_{1} b$ to $r_{2} b$ or vice versa does not change the coset in which $X_{j}$ lies; hence also $T_{1}$ and $N$ are unaffected by changes from $r_{1} b$ to $r_{2} b$ or vice versa in the $\boldsymbol{X}_{\boldsymbol{j}_{i}}-\boldsymbol{X}_{\boldsymbol{j}_{i-1}}$.

Therefore for any subset $I$ of $\mathfrak{S}$

$$
\begin{aligned}
& P_{0}\left[X_{T_{1}} \in I \mid T_{1}=t, N=n, X_{j}-X_{j-1}=r_{1} b \text { or } r_{2} b\right. \text { only for } \\
& \left.\quad j \in\left\{j_{1}, \ldots, j_{n}\right\} \text { and } X_{k}-X_{k-1}=g_{k}, k \notin\left\{j_{1}, \ldots, j_{n}\right\}\right] \\
& =P_{0}\left[\sum_{i=1}^{n}\left(X_{j_{i}}-X_{j_{i}-1}\right) \in I-\sum_{k \notin\left\{j_{1}, \ldots, j_{n}\right\}} g_{k} \mid T_{1}=t, N=n, X_{j}-X_{j-1}=r_{1} b \text { or } r_{2} b\right. \\
& \left.\quad \text { only for } j \in\left\{j_{1}, \ldots, j_{n}\right\} \text { and } X_{k}-X_{k-1}=g_{k}, k=\left\{j_{1}, \ldots, j_{n}\right\}\right] \\
& =P\left[S_{n} \in I-\sum_{k\left\{j_{1}, \ldots, j_{n}\right\}} g_{k}\right],
\end{aligned}
$$

where $S_{n}$ stands for the sum of $n$ independent random variables, each with the distribution (3.18). By theorem 1 of [3] there exists an A such that

$$
P\left[S_{n}=s b\right] \leqslant A n^{-\frac{1}{2}}
$$

for all $s$ and $n \geqslant 1$. Consequently for each set $I$ of fewer than $\frac{1}{2} A n^{\frac{1}{2}}$ points

$$
\begin{aligned}
& P_{0}\left[X_{T_{1}} \in I \mid T_{1}=t, N=n, X_{j}-X_{j-1}=r_{1} b \text { or } r_{2} b\right. \text { only for } \\
& \left.j \in\left\{j_{1}, \ldots, j_{n}\right\} \text { and } X_{k}-X_{k-1}=g_{k}, k \notin\left\{j_{1}, \ldots, j_{n}\right\}\right] \leqslant \frac{1}{2}
\end{aligned}
$$

so that we finally conclude (e.g. from Tchebychev's inequality) that

$$
\begin{aligned}
& \sigma^{2}\left[X_{T_{1}} \mid X_{0}=0, T_{1}=t, N=n, X_{j}-X_{j-1}=r_{1} b \text { or } r_{2} b\right. \text { only for } \\
& \left.\qquad j \in\left\{j_{1}, \ldots, j_{n}\right\} \text { and } X_{k}-X_{k-1}=g_{k}, k \notin\left\{j_{1}, \ldots, j_{n}\right\}\right] \geqslant \frac{A^{2}}{32} n .
\end{aligned}
$$

Thus, for sufficiently small $\varepsilon>0$,

$$
\begin{aligned}
& \sigma^{2}\left[X_{T_{1}} \mid X_{0}=0, T_{1}=t\right] \\
& \geqslant \sum_{\left|n-\left(p_{1}+p_{\xi}\right) t\right| \leqslant \delta t} \sum_{1 \leqslant j_{1}<j_{2} \ldots<j_{n} \leqslant t} \sum_{g_{k}, k \notin\left\{j_{1}, \ldots, j_{n}\right\}} P\left[N=n, X_{j}-X_{j-1}=r_{1} b \text { or } r_{2} b\right. \text { only for } \\
& \left.j \in\left\{j_{1}, \ldots, j_{n}\right\}, X_{k}-X_{k-1}=g_{k}, k \notin\left\{j_{1}, \ldots, j_{n}\right\} \mid X_{0}=0, T_{1}=t\right] . \\
& \sigma^{2}\left[X_{T_{1}} \mid X_{0}=0, T_{1}=t, N=n, X_{j}-X_{j-1}=r_{1} b \text { or } r_{2} b\right. \text { only for } \\
& \left.j \in\left\{j_{1}, \ldots, j_{n}\right\}, X_{k}-X_{k-1}=g_{k}, k \notin\left\{j_{1}, \ldots, j_{n}\right\}\right] \\
& \geqslant \frac{A^{2}}{32} \varepsilon t \cdot P\left[\left|N-\left(p_{1}+p_{2}\right) t\right| \leqslant \varepsilon t \mid X_{0}=0, T_{1}=t\right]
\end{aligned}
$$


Finally, by (3.17)

$$
\begin{aligned}
\sigma^{2}\left[X_{T_{1}} \mid X_{0}=0\right] & \geqslant \sum_{t=1}^{\infty} P_{0}\left[T_{1}=t\right] \sigma^{2}\left[X_{T_{1}} \mid X_{0}=0, T_{1}=t\right] \\
& \left.\geqslant \sum_{t=1}^{\infty} P_{0}\left[T_{1}=t ; \mid N-\left(p_{1}+p_{2}\right) t\right] \leqslant \varepsilon t\right] \frac{A^{2}}{32} \varepsilon t \\
& \geqslant \sum_{t=1}^{\infty}\left\{P_{0}\left[T_{1}=t\right]-C e^{-\lambda t}\right\} \frac{A^{2} \varepsilon t}{32}=\infty
\end{aligned}
$$

since $\sum_{t=1}^{\infty} t P_{0}\left[T_{1}=t\right]$ is the mean recurrence time of the induced random walk on (S) $/ \mathfrak{H}$ (which takes the value $\left\{z_{i}+\mathfrak{H}\right\}$ whenever the original random walk takes a value in the coset $\left.\left\{z_{i}+\mathfrak{S}\right\}\right)$ and any recurrent random walk on an infinite group must be nullrecurrent (cf. comment to (2.9)).

Remark. By a refinement of the above argument we can show that for any aperiodic random walk $X_{0}=0, X_{1}, X_{2}, \ldots$ on the integral points in the plane one has $E_{0}\left[\left|X_{T_{1}}\right|\right]=\infty$ where $X_{T_{1}}$ is the first $X_{n}, n \geqslant 1$ for which the second coordinate is zero.

Before we complete the proof of Theorem 3.1 we remark that (3.7), (3.3) and

$$
\lim _{m \rightarrow \infty} P_{z_{i}+m b}\left[X_{T_{1}} \geqslant k b\right]=\lim _{m \rightarrow \infty} P_{z i}\left[X_{T_{1}} \geqslant(k-m) b\right]=1
$$

for each fixed $k$, immediately imply

$$
\lim _{k \rightarrow \infty} H_{B}\left(z_{i}+k b, 0\right)=\lim _{k \rightarrow \infty} \bar{H}_{B}(k b, 0) .
$$

Similarly

$$
\lim _{k \rightarrow-\infty} H_{B}\left(z_{i}+k b, 0\right)=\lim _{k \rightarrow-\infty} \vec{H}_{B}(k b, 0)
$$

which proves the second equality in (3.6).

Proof of Theorem 3.1. As remarked before, the last equalities in (3.4) and (3.5) follow from Lemmas 3.2 and 3.3. But the existence of $\lim _{|t| \rightarrow \infty} H_{B}(t, 0)$ immediately implies

$$
\lim _{n \rightarrow \infty} \sum_{t \in \mathscr{S}} P_{n+1}(x, t) H_{B}(t, 0)=\lim _{|t| \rightarrow \infty} H_{B}(t, 0)
$$

since

$$
\sum_{t \in \mathbb{B}} P_{n+1}(x, t)=1 \text { and } \lim _{n \rightarrow \infty} P_{n+1}(x, t)=0
$$

for each fixed $t$ (cf. comment to (2.9)). Thus for cases I and II the proof is complete. To prove the first equality in 3.6 in case III, we introduce the random variables

$$
T_{r(n)}=\text { time of the first visit to } \mathfrak{G} \text { after time } n
$$


and

$$
U(n)=U_{r(n)}
$$

Then

$$
\begin{aligned}
\sum_{t \in \mathbb{B}} P_{n+1}(x, t) H_{B}(t, 0) & =\sum_{t \in \mathbb{G}} P_{n+1}(x, t) \sum_{k=-\infty}^{+\infty} P_{t}\left[X_{T_{1}}=k b\right] \bar{H}_{B}(k b, 0) \\
& =\sum_{k=-\infty}^{+\infty} P_{x}[U(n)=k b] \bar{H}_{B}(k b, 0)
\end{aligned}
$$

and it suffices to prove in case III that

$$
\lim _{n \rightarrow \infty} \sum_{k \geqslant k_{0}} P_{z_{i}+m b}[U(n)=k b]=\lim _{n \rightarrow \infty} \sum_{k \geqslant k_{0}-m} P_{z_{i}}[U(n)=k b]=\frac{1}{2}
$$

as well as

$$
\lim _{n \rightarrow \infty} \sum_{k \leqslant k_{0}} P_{z_{i}+m b}[U(n)=k b]=\frac{1}{2}
$$

for each fixed $i, m$ and $k_{0}$.

Since the imbedded random walk $U_{1}, U_{1}+U_{2}, U_{1}+U_{2}+U_{3}, \ldots$ on $\mathfrak{S}$ is recurrent and $\sigma^{2}\left(U_{2}\right)=\sigma^{2}\left(U_{3}\right)=\ldots<\infty$ one necessarily has $E U_{2}=E U_{3}=\ldots=0$. In addition the random variables $T_{k}-T_{k-1}, k=2,3, \ldots$ are identically distributed and

$$
E\left[T_{k}-T_{k-1}\right]=E_{0}\left[T_{1}\right]<\infty
$$

for in case III $\mathbb{B} / \mathfrak{S}$ is finite and thus

$$
P_{x}\left[X_{n} \in \mathfrak{S} \text { for some } n \leqslant n_{0}\right] \geqslant \delta>0
$$

uniformly in $x \in(S)$ for suitable $n_{0}$ and $\delta$. By the strong law of large numbers one has therefore

$$
\frac{r(n)}{n} \rightarrow \frac{1}{E_{0}\left[T_{1}\right]} \text { with probability } 1
$$

and Anscombe's limit theorem [1, Theorem 1] for a random number of summands applies to $U(n)=X_{0}+\sum_{i=1}^{r(n)} U_{i}$. Consequently,

$$
\lim _{n \rightarrow \infty} P_{z_{i}+m b}\left[U(n)=k b \text { with } \frac{k}{\sqrt{n \sigma^{2}\left[\overline{\left.U_{2}\right] / E_{0} T_{1}}\right.}} \leqslant \alpha\right]=\frac{1}{\sqrt{2 \pi}} \int_{-\infty}^{\alpha} e^{-\frac{1}{2} u^{2}} d u,
$$

which implies (3.25) and (3.26). The proof of Theorem 3.1 is complete in all cases.

From (2.5) and Theorem 3.1 we immediately derive

ThEOREM 3.2. $a(b)=\lim _{n \rightarrow \infty} a_{n}(b)$ exists and is given by

$$
a(b)=\frac{1}{\prod_{B}(b, 0)} \lim _{|x| \rightarrow \infty} H_{B}(x, 0)
$$


in cases I and II. In case III

for each $i$.

$$
a(b)=\frac{1}{2 \prod_{B}(b, 0)} \lim _{k \rightarrow \infty}\left[H_{B}\left(z_{i}+k b, 0\right)+H_{B}\left(z_{i}-k b, 0\right)\right]
$$

The asymptotic behavior of $a(x)-a(x-b)(|x| \rightarrow \infty)$ is now derived very easily from (2.3) and Theorems 3.1 and 3.2.

THEOREM 3.3. In cases I and II

whereas in case III

$$
\lim _{|x| \rightarrow \infty}[a(x)-a(x-b)]=0
$$

$$
\begin{aligned}
& \lim _{k \rightarrow \infty}\left[a\left(z_{i}+k b\right)-a\left(z_{i}+(k-1) b\right)\right]=\frac{1}{\sigma^{2}\left[U_{2}\right]} \\
\text { and } \quad \lim _{k \rightarrow-\infty}\left[a\left(z_{i}+k b\right)-a\left(z_{i}+(k-1) b\right)\right] & =-\frac{1}{\sigma^{2}\left[U_{2}\right]} .
\end{aligned}
$$

Moreover, in case III, for every $x, y \in \mathbb{S}$ the two limits

$$
\lim _{k \rightarrow \pm \infty}[a(x+y+k b)-a(x+k b)]
$$

exist and are independent of $x$.

Proof. In the notation of (3.1), we have by taking limits $(n \rightarrow \infty)$ in (2.3)

$$
\varphi-H_{B}(x, 0)=[a(x)-a(x-b)] \prod_{B}(b, 0) .
$$

In cases $I$ and II, $\varphi=\lim _{|x| \rightarrow \infty} H_{B}(x, 0)$ according to (3.4) and (3.5), from which (3.27) follows since $\prod_{B}(b, 0)>0$ for an aperiodic random walk. In case III we substitute $z_{i}+k b$ for $x$ in (3.31) and let $k \rightarrow \infty$. From (3.6) and (3.23) one obtains

$$
\frac{1}{2 \prod_{B}(b, 0)} \lim _{k \rightarrow \infty}\left[\bar{H}_{B}(-k b, 0)-\bar{H}_{B}(k b, 0)\right]=\lim _{k \rightarrow \infty}\left[a\left(z_{i}+k b\right)-a\left(z_{i}+(k-1) b\right)\right],
$$

where the existence of the limit in the right-hand side is part of the conclusion. The left-hand side is independent of $i$ and actually depends on the imbedded random walk only. Since every argument remains valid in the special case where $\mathfrak{G}=\mathfrak{H}$ and the original random walk is the same as the imbedded random walk on 5 , the left-hand side of (3.32) must also equal 


$$
\lim _{k \rightarrow \infty}[\bar{a}(k b)-\bar{a}((k-1) b)]
$$

where $\bar{a}$ is the potential kernel for the imbedded random walk. But (3.33) is known (T 29.1 in $[10])$ to have the value $1 / \sigma^{2}\left[U_{2}\right]$ so that for each fixed $i$

$$
\lim _{k \rightarrow \infty}\left[a\left(z_{i}+k b\right)-a\left(z_{i}+(k-1) b\right)\right]=\lim _{k \rightarrow \infty}[\bar{a}(k b)-\bar{a}((k-1) b)]=\frac{1}{\sigma^{2}\left[U_{2}\right]}
$$

and (3.29) is proved similarly.

As for (3.30), for the existence of the limits it suffices to prove the existence of

$$
\lim _{k \rightarrow \pm \infty}\left[a\left(z_{i}+k b\right)-a(k b)\right]
$$

since $x$ and $x+y$ are of the form $z_{i}+m b$ and (3.28) and (3.29) have been proved already. Now $h z_{i}=k_{0} b$ for some finite $h$ and $k_{0}$ since $\left(\mathfrak{S} / \mathfrak{H}\right.$ is finite. If $k_{0}=0$ then $z_{i}$ has finite order in (G) and (3.34) exists by (3.27) with $z_{i}$ taking the place of $b$. If $k_{0} \neq 0$ then each $k$ can be written as $r k_{0}+s, 0 \leqslant s \leqslant k_{0}-1$ and

$$
a\left(z_{i}+k b\right)-a(k b)=a\left(z_{i}+r h z_{i}+s b\right)-a\left(r h z_{i}+s b\right)=a\left(z_{i}+r h z_{i}\right)-a\left(r h z_{i}\right)+o(1) \quad(|k| \rightarrow \infty)
$$

by (3.28) and (3.29). But $\lim _{r \rightarrow \pm \infty} a\left(z_{i}+r h z_{i}\right)-a\left(r h z_{i}\right)$ exists by (3.27)-(3.29), again with $z_{i}$ taking the place of $b$. This proves the existence of (3.34) in all cases and one could even use the above argument to evaluate the limits (3.30) more explicitly to show their independence of $x$. It is easier, however, to recall that

$$
g_{0}(u, v)=E_{u}[\text { number of visits to } v \text { before entering } 0]=a(u)+a(-v)-a(u-v)
$$

(cf. [10] P 11.6 and $P$ 29.4, the proof given there remains valid for arbitrary (S)) so that $a(x+y+k b)-a(x+k b)=g_{0}(x+y+k b, y)-a(-y)$. Thus also

$$
\lim _{k \rightarrow \pm \infty} g_{0}(x+y+k b, y)
$$

exist and we only have to show that these are independent of $x$. The interpretation of $g_{0}(\cdot, \cdot)$ as an expectation implies immediately

so that

$$
g_{0}\left(x_{1}, y\right) \geqslant P_{x_{1}}\left[\text { visit } x_{2} \text { before } 0\right] g_{0}\left(x_{2}, y\right)
$$

$$
g_{0}\left(x_{1}+y+k b, y\right) \geqslant P_{x_{1}+y+k b}\left[\text { visit } x_{2}+y+k b \text { before } 0\right] g_{0}\left(x_{2}+y+k b, y\right) \text {. }
$$

Since the random walk is recurrent

$$
\lim _{k \rightarrow \pm \infty} P_{x_{1}+y+k b}\left[\text { visit } x_{2}+y+k b \text { before } 0\right]=1
$$

which shows that $\lim _{k \rightarrow \pm \infty} g_{0}(x+y+k b, y)$ is the same for all $x$. 
Remark. In a different terminology, (3.27) and the existence and independence of $x$ of the limits (3.30) can be expressed as follows: The Martin boundary for the random walk restricted to $\mathbb{S}-\{0\}$ consists of one point only if case I or II applies for every element $b \in(\mathfrak{S}$, and of two points if case III applies for some $b \in(\mathfrak{S}$. The regular functions of this random walk will be determined in the next section, following the proof of Theorem 4.5.

\section{Asymptotic behavior of potential kernels}

This section is devoted to both recurrent and transient random walk on a count. ably infinite Abelian group (65. As heretofore the underlying measure $\mu$ is assumed to be aperiodic. When $\mu$ is transient, it is obvious that the potential kernel $G(x, y)$ satisfies

$$
G(x, y)-\sum_{t \in \mathbb{G}} P(x, t) G(t, y)=\delta(x, y), \quad x, y \in \mathbb{G} \text {. }
$$

In the recurrent case, however, the proof that the potential kernel $A(x, y)$ (which exists according to Theorem 3.2 ) satisfies

$$
\sum_{i \in \mathbb{S}} P(x, t) A(t, y)-A(x, y)=\delta(x, y), \quad x, y \in \mathbb{B}
$$

is somewhat more delicate; however, the proof of $P$ 13.3 of [10] applies to arbitrary (S) with obvious modifications. We now proceed to develop certain results concerning the kernels $G$ and $A$ which depend to some extent on (4.1) and (4.2) and which exhibit strong similarity-so strong in fact that we suspect this similarity is only partly explained by the formal similarity between the Poisson type equations (4.1) and (4.2).

THEOREM 4.1 (a). Suppose $\mu$ is transient. There are then two possibilities. Either $g(x)=G(x, 0)$ tends to zero as $|x| \rightarrow \infty$ or it does not. In the latter, case there is exactly one number $L>0$ for which there exist sequences $x_{n} \in(S)$ such that $g\left(x_{n}\right) \rightarrow L$ and $\left|x_{n}\right| \rightarrow \infty$. Moreover, given any infinite subset $S \subset \mathbb{S}$, there exists a sequence of points $y_{n} \in S$ such that $\left|y_{n}\right| \rightarrow \infty$ and such that either

or

$$
\lim _{n \rightarrow \infty} g\left(y_{n}\right)=0 \text { and } \lim _{n \rightarrow \infty} g\left(-y_{n}\right)=\mathrm{L}
$$

If, in particular $S$ is the infinite cyclic subgroup

$$
\lim _{n \rightarrow \infty} g\left(y_{n}\right)=L \text { and } \lim _{n \rightarrow \infty} g\left(-y_{n}\right)=0 \text {. }
$$




$$
\mathfrak{S}=\{n z\}, n=0, \pm 1, \ldots, z \in \mathbb{S},
$$

then we may choose $y_{n}=n z, n \geqslant 1$.

THEOREM 4.1. (b). Suppose that $\mu$ is recurrent. There are then two possibilities. Either $a(x)=A(x, 0)$ tends to infinity as $|x| \rightarrow \infty$ or it does not. In the latter case there is exactly one number $L<\infty$ (it is non-negative but may be zero) for which there exist sequences $x_{n} \in \mathbb{S}^{\prime}$ such that $g\left(x_{n}\right) \rightarrow L$ and $\left|x_{n}\right| \rightarrow \infty$. The rest of the theorem reads just as part (a), except that the limit 0 is replaced by $\infty$.

The proof of part (a) is an immediate consequence of four familiar properties of the potential kernel $g(x)=G(x, 0)$.

$$
\begin{gathered}
0 \leqslant g(x) \leqslant g(0), \quad x \in \mathbb{B} \\
\lim _{|x| \rightarrow \infty}[g(x)+g(-x)]=L \text { exists } \\
\lim _{|x| \rightarrow \infty}[g(x+y)-g(x)]=0, \quad y \in \mathbb{G}, \\
\lim _{|x| \rightarrow \infty} g(x) g(-x)=0 .
\end{gathered}
$$

These are all well known when $\left(\mathfrak{S}=Z_{d} .(4.3)\right.$ is obvious and the other three are due to Feller and Orey (see [7]). Their proofs as well as those in [10] extend to general (S) without difficulty. In particular the proof of (4.4) rests on an application of the Riemann Lebesgue lemma to the representation of $g(x)+g(-x)$ as an integral over the character group $\Gamma$. This causes no concern, and neither does (4.5) which depends on the lemma of Choquet and Deny mentioned in connection with equation (2.7).

If the constant $L$ in (4.4) is zero then we are in the first case of Theorem 4.1 (a), and there is nothing to prove. If $L>0$ it follows from (4.3) that $g$ is bounded, and from (4.4) and (4.6) that $\left|x_{n}\right| \rightarrow \infty, g\left(x_{n}\right) \rightarrow M>0$ implies $M=L$ and $\lim _{n \rightarrow \infty} g\left(-x_{n}\right)=0$. When $S$ is an unspecified infinite subset, choose any sequence $x_{n} \in S$ such that $\left|x_{n}\right| \rightarrow \infty$. $\mathrm{Bx}$ (4.4) we can select a subsequence $y_{n}$ such that either $\lim g\left(y_{n}\right)>0$ or $\lim g\left(-y_{n}\right)>0$. But we showed that such a positive limit must have the value $L$, and that the limit of the sequence whose sign is reversed must be zero. Finally, when $S=\mathfrak{g}=\{n z\}$ we invoke (4.5) with $y=z$ which prevents the oscillation which would occur if $0=$ $\lim \inf g(n z)<\lim \sup g(n z)=L$ (in this case every point in $[0, \mathrm{~L}]$ would be an accumulation point of $g(n z)$, which is impossible).

The proof of Theorem 4.1 (b) is based on the inequality $17-652933$ Acta mathematica 114. Imprimé le 15 octobre 1965. 


$$
[a(-y)-a(x-y)] a(y) \leqslant a(x) a(-y), \quad x, y \in(\mathfrak{S}
$$

which we proceed to derive. We shall first assume that neither $x$ nor $y$ belong to the set $N=[x \mid a(x)=0]$. (Note that $a(0)=0$ so that $N$ is nonempty, but that $N$ may be much larger, as shown in [10], $P$ 30.2.) We denote

$$
\begin{aligned}
& Q^{0}(x, y)=P(x, y), \quad x, y \in \mathbb{S}-\{0\} \\
& Q^{N}(x, y)=P(x, y), \quad x, y \in \mathbb{S}-N
\end{aligned}
$$

i.e. the transition functions restricted to the complement of the origin and of $N$, and $Q_{n}^{0}$ and $Q_{n}^{N}$ will denote the iterates of $Q^{0}$ and $Q^{N}$. It follows from (4.2) that

$$
\sum_{y \in\{0\}} Q_{n}^{0}(x, y) a(y)=a(x), \quad x \in(S \mathcal{S}-\{0\}, n \geqslant 0 .
$$

This implies that, when $x \in N-\{0\}, Q_{n}^{0}(x, y)=0$ for all $y \in \mathbb{G}-N$. Consequently the random walk can only leave the set $N-\{0\}$ by going to 0 . But that may be expressed, in terms of the Green functions

by saying that

$$
g_{0}(x, y)=\sum_{n=0}^{\infty} Q_{n}^{0}(x, y), g_{N}(x, y)=\sum_{n=0}^{\infty} Q_{n}^{N}(x, y)
$$

$$
g_{0}(x, y)=g_{N}(x, y), \quad x, y \in \mathbb{S}-N
$$

Also it is known (cf. (3.35)) that

$$
g_{0}(x, y)=a(x)+a(-y)-a(x-y), \quad x, y \in \mathfrak{G}
$$

Observe now, using (4.2), that the transient Markov chain with state space $B-N$ and transition function

$$
P^{N}(x, y)=\frac{Q^{N}(x, y) a(y)}{a(x)}, \quad x, y \in \mathbb{S}-N
$$

has the Green function

$$
G_{N}(x, y)=\sum_{n=0}^{\infty} P_{n}^{N}(x, y)=\frac{a(y)}{a(x)} \sum_{n=0}^{\infty} Q_{n}^{N}(x, y)=\frac{a(y)}{a(x)} g_{N}(x, y), \quad x, y \in(\mathfrak{S}-N
$$

Here $P_{n}^{N}$ denotes the iterates ( $n$-step transition functions) of $P^{N}$. From the maximum principle of potential theory in its probabilistically obvious form

$$
G_{N}(x, y) \leqslant G_{N}(y, y)
$$

we conclude by combining (4.8), (4.9), and (4.10) that 


$$
\frac{a(y)}{a(x)}[a(x)+a(-y)-a(x-y)] \leqslant a(y)+a(-y), \quad x, y \in(\mathfrak{H}-N
$$

When simplified, this is exactly the inequality (4.7). When $a(y)=0$ then (4.7) is obvious. When $x=0$ it is also clear. Assume finally that $x \in N-\{0\}$ but $a(y) \neq 0$. Then, $y \in(S)-N$ and as remarked in the derivation of $(4.8), Q_{n}^{0}(x, y)=0$ for all $n \geqslant 0$ so that $g_{0}(x, y)=0$, and by $(4.9)$

$$
g_{0}(x, y)=a(-y)-a(x-y)=0
$$

That completes the proof of the inequality (4.7).

Proceeding with the proof of Theorem 4.1 (b) we may assume that $a(x)$ does not tend to infinity as $|x| \rightarrow \infty$. Suppose further that $\left|x_{n}\right| \rightarrow \infty$ and $a\left(x_{n}\right) \rightarrow L<\infty$. Then we know that $a\left(-x_{n}\right) \rightarrow \infty$, since as pointed out in the proof of Theorem $2.1 S(x)=$ $a(x)+a(-x) \rightarrow \infty$ as $|x| \rightarrow \infty$. Setting $y=-x_{n}$ in (4.7) one readily obtains

$$
\limsup _{n \rightarrow \infty}\left[a\left(x_{n}\right)-a\left(x+x_{n}\right)\right] \leqslant 0
$$

To show that in fact the limit of $a\left(x_{n}\right)-a\left(x+x_{n}\right)$ exists and is zero, observe, say by use of (3.31), that $a\left(x_{n}-x\right)$ in a bounded sequence when $a\left(x_{n}\right)$ in bounded. Therefore $a\left(x-x_{n}\right) \rightarrow \infty$. For this reason we may set $y=-x_{n}+x$ in $(4.7)$, let $n \rightarrow \infty$, and conclude that

$$
\limsup _{n \rightarrow \infty}\left[a\left(x_{n}-x\right)-a\left(x_{n}\right)\right] \leqslant 0
$$

Since $-x$ may be replaced by $x$ we have shown that

$$
\lim _{n \rightarrow \infty}\left[a\left(x_{n}\right)-a\left(x_{n}+x\right)\right]=0
$$

whenever $\left|x_{n}\right| \rightarrow \infty$ and $a\left(x_{n}\right) \rightarrow L<\infty$.

In order to establish the uniqueness of $L$ we now suppose that

$$
\left|x_{n}\right| \rightarrow \infty,\left|y_{n}\right| \rightarrow \infty, a\left(x_{n}\right) \rightarrow L<\infty, a\left(y_{n}\right) \rightarrow M<\infty,
$$

and employ (4.7) together with (4.11) to prove that $M=L$. By (4.11)

$$
\lim _{n \rightarrow \infty}\left[a(-y)-a\left(x_{n}-y\right)\right] a(y)=\lim _{n \rightarrow \infty}\left[a(-y)-a\left(x_{n}\right)\right] a(y)=[a(-y)-L] a(y),
$$

but from (4.7) we have the inequality

$$
[a(-y)-L] a(y) \leqslant L a(-y)
$$


into which we substitute $y=-y_{n}$, and let $n \rightarrow \infty$ to obtain

$$
(M-L) \lim _{n \rightarrow \infty} a\left(-y_{n}\right) \leqslant L M
$$

Since $a\left(-y_{n}\right) \rightarrow \infty$ it follows that $L \geqslant M$, but as there is nothing to distinguish $L$ and $M$ we have proved that $L=M$.

To establish the asymptotic behavior of the potential kernel on an infinite subset $S \subset(\mathfrak{S})$ we first take for $x_{n}$ any sequence in $(S)$ such that $\left|x_{n}\right| \rightarrow \infty$ and $a\left(x_{n}\right) \rightarrow L$, Then, from (4.12)

$$
a(y) a(-y) \leqslant L[a(y)+a(-y)] \leqslant 2 L \max \{a(y), a(-y)\}
$$

Thus for each $y \in(S)$

$$
\text { either } a(y) \leqslant 2 L \text { or } a(-y) \leqslant 2 L \text {. }
$$

Now the rest of the proof of Theorem $4.1(\mathrm{~b})$ is ovious. Either $a(y) \rightarrow \infty$ as $|y| \rightarrow \infty$ in $S$, in which case any subsequence $y_{n}$ of $S$ with $\left|y_{n}\right| \rightarrow \infty$ has the property that $a\left(y_{n}\right) \rightarrow \infty$, and then (4.13) and the first part of the theorem guarantees that $a\left(-y_{n}\right) \rightarrow L$. Or else $S$ contains a subsequence $x_{n}$ such that $\left|x_{n}\right| \rightarrow \infty$ and $\lim \sup a\left(x_{n}\right)<\infty$. Then by the first part of the theorem $x_{n}$ has a subsequence $y_{n}$ such that $a\left(y_{n}\right) \rightarrow L$, and since $a(x)+a(-x)$ tends to infinity with $|x|$ we have $a\left(-y_{n}\right) \rightarrow \infty$. It only remains to consider the special case when $S=\mathfrak{S}=\{n z\}$. The sequence $x_{n}=n z, n \geqslant 1$ is then seen to have the desired behavior by using (4.11) in just the same way that (4.5) was used in the proof of Theorem 4.1 (a). The proof of Theorem 4.1 is now complete.

THEOREM 4.2. Suppose that the group (S) has an infinite cyclic subgroup $\mathfrak{H}$ such that $(\mathbb{S} / \mathfrak{S}$ is infinite, or that $(S)$ has only elements of finite order. Then every transient random walk on (S) has the property that $g(x)=G(x, 0) \rightarrow 0$ as $|x| \rightarrow \infty$, and for every recurrent random walk $a(x)=A(x, 0) \rightarrow \infty$, as $|x| \rightarrow \infty$.

Proof. The proof is simplest in the first case. Then for some $y \in(B)$, of infinite order, and $\mathfrak{H}=\{n y\}, n=0, \pm 1, \ldots, \mathfrak{S} / \mathfrak{G}$ is infinite. We argue by contradiction and suppose in the transient case that $g(x) \rightarrow L>0$ along some sequence tending to infinity, and in the recurrent case that $a(x) \rightarrow L<\infty$. In the transient case it follows from Theorem 4.1 (a) that there exists a subsequence $z_{n}^{\prime}$ of the representatives $z_{k}$ of the cosets of $\mathfrak{f}$, such that either $g\left(z_{n}^{\prime}\right) \rightarrow L$ and $g\left(-z_{n}^{\prime}\right) \rightarrow 0$ or such that the limits are reversed. We assume without loss of generality that the former alternative holds. Further, by the last part of Theorem 4.1 (a) either $g(k y) \rightarrow 0$ and $g(-k y) \rightarrow L$ or the limits are reversed. Again without loss of generality (as is clear from the rest of the 
proof) assume the former contingency. It follows from equation (4.5) that $g\left(z_{n}^{\prime}+k y\right)$ for fixed $k$ tends to $L>0$ as $n \rightarrow \infty$ and for fixed $n$ to 0 as $k \rightarrow \infty$. For all sufficiently large values of $n \geqslant 1$ we may therefore pick the largest $k=k_{n}$ such that $g\left(z_{n}^{\prime}+k_{n} y\right)>\frac{1}{2} L$. Then $g\left(z_{n}^{\prime}+k_{n} y+y\right) \leqslant \frac{1}{2} L$, and it follows from Theorem 4.1 that

$$
\lim _{n \rightarrow \infty} g\left(z_{n}^{\prime}+k_{n} y\right)=L>0, \lim _{n \rightarrow \infty} g\left(z_{n}^{\prime}+k_{n} y+y\right)=0 .
$$

This is in contradiction to equation (4.5). In the recurrent case we arrive at a contradiction in exactly the same fashion, using (4.11) in place of (4.5).

The second half of the proof, concerning groups $(S)$ which have only elements of finite order, is more complicated. We shall give the details in the recurrent case. Assuming again that $a(x)$ does not tend to infinity as $|x| \rightarrow \infty$ we may select a sequence $z_{n}$ of distinct elements, whose orders are $h_{n}>1$, such that $a\left(z_{n}\right) \rightarrow L<\infty$ as $n \rightarrow \infty$. Then, observing that $\left(h_{n}-1\right) z_{n}=-z_{n}$, we see from Theorem 4.1 (b) that

$$
a\left[\left(h_{n}-1\right) z_{n}\right] \rightarrow \infty
$$

(This shows, by the way that $h_{n}>2$.) At this point we may select, at least for large enough $n$, an integer $k_{n}$ with the property of being the largest positive integer less than $h_{n}-1$, such that $a\left(k_{n} z_{n}\right) \leqslant 4 L+4$. From equation (4.9) we have

$$
g_{0}(x, y)=a(x)+a(-y)-a(x-y) \geqslant 0
$$

which we shall use in the form

$$
a\left[\left(k_{n}+1\right) z_{n}\right]-a\left(k_{n} z_{n}\left(z_{n}\right)\right) \leqslant a_{n} \leqslant 2 L+2
$$

when $n$ is sufficiently large. It follows from the definition of $k_{n}$ and (4.14) that

$$
2 L+2 \leqslant a\left(k_{n} z_{n}\right) \leqslant 4 L+4 \leqslant a\left[\left(k_{n}+1\right) z_{n}\right] \leqslant 6 L+6 .
$$

If we knew that the sequence $k_{n} z_{n}$ contains infinitely many distinct elements of (S) then we would have a contradiction (on a subsequence of this sequence $a(x)$ would then converge to a number between $2 L+2$ and $4 L+4$ which contradicts Theorem $4.1(b))$. Indeed there is no way of knowing whether $\left\{k_{n} z_{n}\right\}$ is of infinite cardinality, but if not, then $\left\{\left(k_{n}+1\right) z_{n}\right\}$ is of infinite cardinality, since $\left\{z_{n}\right\}$ contains infinitely many distinct elements. In that case the desired contradiction comes from the third and fourth inequalities.

The proof in the transient case is very similar. We have $g\left(z_{n}\right) \rightarrow L, g\left[\left(h_{n}-1\right) z_{n}\right] \rightarrow 0$, and define $k_{n}$ as the largest integer less than $h_{n}-1$ such that $g\left(k_{n} z_{n}\right) \geqslant L^{2} /(4 g(0))$. 
(This is possible since $L \leqslant g(0)$.) The analogue of (4.14) comes from the elementary inequality

$$
g(x-y) g(y) \leqslant g(0) g(x)
$$

which gives

$$
\frac{g\left[\left(k_{n}+1\right) z_{n}\right]}{g\left(k_{n} z_{n}\right)} \geqslant \frac{g\left(z_{n}\right)}{g(0)} \geqslant \frac{L}{2 g(0)}
$$

It follows that

$$
\frac{L}{2} \geqslant g\left(k_{n} z_{n}\right) \geqslant \frac{L^{2}}{4} g(0) \geqslant g\left[\left(k_{n}+1\right) z_{n}\right] \geqslant \frac{L^{3}}{8[g(0)]^{2}}>0
$$

Again Theorem 4.1 leads to the desired contradiction.

Up till now, in Theorems 4.1 and 4.2 we were concerned with general assertions concerning the asymptotic behavior of the potential kernels-assertions which depended on the group structure, but not on the given probability measure $\mu$. The next theorem gives a criterion which does depend on $\mu$ (but in a rather complicated way) for whether $g(x) \rightarrow 0$ as $|x| \rightarrow \infty$ or not. This criterion will then be simplified in Theorem 4.4.

THEOREM 4.3. Consider transient random walk on a group (G) with an infinite cyclic subgroup $\mathfrak{H}=\{n y\}$ such that $\mathfrak{B} / \mathfrak{S}$ is finite. Then the statement (a): $g(x)$ does not tend to zero as $|x| \rightarrow \infty$ is equivalent to (b): the imbedded random walk $X_{T_{k}}, k \geqslant 1$, starting at $X_{0}=0$ (defined at the beginning of section 3) has finite non-zero mean (in the sense that $\sum_{k=-\infty}^{+\infty} P_{0}\left[X_{T_{1}}=k y\right] k$ is absolutely convergent and non-zero).

Proof. Suppose first that (b) holds. Let $\bar{G}(x, y)$ denote the Green function of the imbedded random walk, which is a one-dimensional random walk on

$$
\mathfrak{S}=\{n y\}, n=0, \pm 1, \ldots
$$

By the renewal theorem for one-dimensional random walk ([10], T 24.2) $\bar{G}(0, n y)$ tends to a positive limit (the reciprocal of the absolute value of the mean of the random walk $\left.X_{T_{k}}\right)$ either as $n \rightarrow+\infty$ or as $n \rightarrow-\infty$. On the other hand $\bar{G}(0, x)=G(0, x)$ when $x \in \mathfrak{S}$, which proves that (a) holds.

To go the other way suppose that (b) is false. Let $\bar{G}(x, 0)=\bar{g}(x), G(x, 0)=g(x)$, as usual. The renewal theorem now gives $\bar{g}(n y)=g(n y) \rightarrow 0$ as $n \rightarrow \pm \infty$. Therefore the last statement of Theorem 4.1 (a) implies that

$$
g(x) \rightarrow 0 \text {, as }|x| \rightarrow \infty \text { in } \mathbb{S} \text {. }
$$

Thus (a) is false, and Theorem 4.3 is proved. 
Our final results depend on a special case of a theorem of Kaplansky concerning homomorphisms of Abelian groups (see [9], p. 44).

LEMMA 4.1. Let $\mathbb{S}$ be infinite Abelian, and $\mathfrak{H}=\{n y\}$ an infinite cyclic subgroup. Suppose that $(\mathfrak{S} / \mathfrak{S}$ is finite. The isomorphism $n y \rightarrow n$ of $\mathfrak{g}$ onto the integers can then be extended, in one and only one way, to a homomorphism $\psi: \mathfrak{G} \rightarrow \Re$, the additive group of rationals.

Observe that $\psi$ is quite explicitly known. When $x \in \mathfrak{H} \psi(x)=n$, where $x=n y$; when $x \notin \tilde{D}$ observe that $n x \in \mathfrak{H}$ for some integer $n$ (since $\mathfrak{S} / \mathfrak{H}$ is finite); then $n x=m y$ and $\psi(n x)=n \psi(x)=m$.

THEOREM 4.4. For a transient random walk the statements (a) and (b) in Theorem 4.3 are equivalent to

$$
\sum_{x \in \mathscr{S}} P(0, x)|\psi(x)|<\infty \text { and } \sum_{x \in \mathbb{G}} P(0, x) \psi(x) \neq 0
$$

Proof. We shall prove the equivalence of (b) and (c). We let $Z_{n}=\psi\left(X_{n}\right)$, where $X_{n}$ is the given transient random walk on $\left(\mathcal{G}\right.$, and observe that $Z_{n}$ is a random walk on the subgroup $\psi(\mathfrak{G})$ of $\mathfrak{R}$. The sums in (c) above are simply the moments $E_{0}\left[\left|Z_{1}\right|\right]$ and $E_{0}\left[Z_{1}\right]$.

Suppose now that (b) holds. If $\mathfrak{G}=\bigcup_{i=0}^{p}\left\{\mathfrak{H}+z_{i}\right\}, z_{0}=0$, then

$$
E_{0}\left[\left|Z_{1}\right|\right]=\sum_{i=0}^{p} \sum_{k=-\infty}^{\infty} P\left(0, k y+z_{i}\right)\left|k+\psi\left(z_{i}\right)\right|
$$

and therefore $E_{0}\left|Z_{1}\right|$ will be finite if we show that

$$
\sum_{k=-\infty}^{\infty} P\left(0, k y+z_{i}\right)|k|<\infty, \text { for } i=0,1, \ldots, p
$$

The imbedded random walk $X_{T_{n}}=U_{1}+\ldots+U_{n}, n \geqslant 0$, with $X_{0}=0$ satisfies

$$
\sum_{k=-\infty}^{+\infty} P_{0}\left[X_{T_{1}}=k y\right]|k|=E_{0}\left|\psi\left(U_{1}\right)\right| \geqslant \sum_{-\infty}^{\infty} P(0, k y)|k|
$$

so that (4.18) holds for $z_{i}=z_{0}=0$. For every other $z_{i}$ we can find integers $n \geqslant 1$, and $-\infty<m<\infty$ such that

$$
P_{0}\left[X_{n}=m y-z_{i} ; X_{k} \notin \mathfrak{S} \text { for } 1 \leqslant k<n\right]=\alpha_{i}>0 \text {. }
$$

Then $\quad E_{\mathbf{0}}\left|\psi\left(U_{1}\right)\right| \geqslant \alpha_{i} \sum_{k=-\infty}^{\infty} P\left(m y-z_{i}, k y\right)|k|=\alpha_{i} \sum_{k=-\infty}^{+\infty} P\left(0, k y+z_{i}\right)|k+m|$, 
and (4.19) implies that the sums in (4.18) are finite. Thus (b) implies that $E_{0}\left[\left|Z_{1}\right|\right]<\infty$. To show that $E_{0}\left[Z_{1}\right] \neq 0$ we use the strong law of large numbers. It asserts that

$$
\lim _{n \rightarrow \infty} \frac{Z_{n}}{n}=E_{0}\left[Z_{1}\right]
$$

Since $\psi\left(\boldsymbol{X}_{T_{k}}\right)$ is a (random) subsequence of $Z_{n}$, we have also

$$
\lim _{k \rightarrow \infty} \frac{\psi\left(X_{T_{k}}\right)}{T_{k}}=E_{0}\left[Z_{1}\right]
$$

the random variables $T_{n}, n \geqslant 1$, being the times of successive visits of $X_{n}$ to $\mathfrak{G}$. But by assumption (b) we may apply the strong law of large numbers directly to the imbedded random walk to obtain

$$
\lim _{k \rightarrow \infty} \frac{\psi\left(X_{T_{k}}\right)}{k}=\lim _{k \rightarrow \infty} \frac{\sum_{n=1}^{k} \psi\left(U_{n}\right)}{k}=E_{0}\left[\psi\left(U_{1}\right)\right] \neq 0 .
$$

Finally

$$
\lim _{n \rightarrow \infty} \frac{T_{n}}{n}=E_{0}\left[T_{1}\right]<\infty
$$

and this is the mean recurrence time of the induced random walk on the finite group (J) $/ \mathfrak{S}$. Combining $(4.20)-(4.23)$ it is clear that $E_{0}\left(Z_{1}\right) \neq 0$ which completes the proof of statement (c).

To prove the converse we assume (c). Then (4.20) and hence (4.21) hold with $E_{0}\left[Z_{1}\right] \neq 0,(4.23)$ is also valid, and henee the limit

$$
\lim _{k \rightarrow \infty} \frac{\psi\left(X_{T_{k}}\right)}{k}=\lim _{k \rightarrow \infty} \frac{\psi\left(X_{T_{k}}\right)}{T_{k}} \lim _{n \rightarrow \infty} \frac{T_{n}}{n}
$$

exists and is non-zero. It therefore follows from the converse of the strong law of large numbers that $\psi\left(X_{T_{1}}\right)=\psi\left(U_{1}\right)$ has a finite non-zero mean, which completes the proof of Theorem 4.4.

Remark. Statement (c) in Theorem 4.4 seems to depend on the generator $y$ of the cyclic group $\mathfrak{H}$, since the construction of the homomorphism $\psi=\psi_{y}$ of $\mathfrak{B S}$ into $\mathfrak{R}$ depended on $y$. In fact (c) is independent of $y$, in the sense that it holds, or fails, simultaneously for all elements $y$ of infinite order. This is easily verified, as elementary group theoretical arguments show: 
(i) if $y$ and $y^{\prime}$ are elements of infinite order, generating the subgroups $\mathfrak{S}$ and $\mathbb{S}_{3}^{\prime}$, and if $\mathfrak{G S} / \mathfrak{S}$ is finite, then so is $\mathscr{G} / \mathfrak{S}^{\prime}$.

(ii) under the assumptions in (i) the homomorphisms $\psi$ and $\psi^{\prime}$ of $\mathfrak{\mathfrak { g }}$ and $\mathfrak{S}^{\prime}$ into $\Re$ of Lemma 4.1 satisfy $\psi^{\prime}(x)=c \psi(x), x \in \mathfrak{B}$, for some non-zero constant $c$.

There is at present no analogue for recurrent random walk of Theorems 4.3 and 4.4. The reason is that even when $\mathbb{B}=Z_{1}$ (the integers) we have no satisfactory necessary and sufficient condition on the measure $\mu$ under which $a(x)=A(x, 0)$ fails to tend to infinity as $|x| \rightarrow \infty$. Examples of this phenomenon are furnished, however, by all recurrent random walks on $Z_{1}$ such that

$$
\sum_{-\infty}^{\infty} n^{2} \mu(n)=\infty \text { and } \sum_{n=M}^{\infty} \mu(n)=0 \text { for some } M>0 .
$$

If such a condition were known on $Z_{1}$, then presumably the method of proof of Theorem 4.4 would lead to its generalization for arbitrary (SF. Instead we apply this method to a different problem concerning recurrent random walk.

THEOREM 4.5. For a recurrent random walk the following three conditions are equivalent.

(a) The random walk is of type III with respect to some group element $y$ of infinite order, i.e. the factor group $\mathfrak{H} / \mathfrak{H}$, where $\mathfrak{H}=\{n y\}$, is of finite order, and the imbedded random walk on $\mathfrak{S}$ has finite variance.

(b) There exists some $z \in(S)$ such that $a(x+z)-a(x)$ does not tend to 0 as $|x| \rightarrow \infty$.

(c) For some $y$ of infinite order, and $\mathfrak{H}=\{n y\}$, the factor group $\mathbb{S} / \mathfrak{S}$ is finite, and the homomorphism in $\psi=\psi_{y}$ of Lemma 4.1 satisfies

$$
\sum_{x \in \mathbb{S}} P(0, x) \psi(x)=0, \quad \sum_{x \in \mathbb{S}} P(0, x) \psi^{2}(x)<\infty
$$

We sketch the proof, which involves no new ideas. By remarks (i) and (ii) following the proof of Theorem 4.4 statement (c) will hold simultaneously for all $y$ of infinite order, if it holds for one of them. Therefore (a) will be equivalent to (c) if we prove the equivalence for a fixed element $y$ of infinite order. Then we shall know that a recurrent random walk is of type III either with respect to all elements of infinite order, or with respect to none, and consequently Theorem 3.3 shows that (a) is equivalent to (b).

Suppose therefore that (a) holds for some infinite cyclic group $\mathfrak{S}=\{n y\}$. Then it 
may be shown (by exactly the same method as in equation (4.19) of the proof of Theorem 4.4) that

$$
E_{0}\left[\psi\left(X_{1}\right)^{2}\right]=\sum_{x \in \mathbb{G}} P(0, x) \psi^{2}(x)<\infty .
$$

It is also clear that the random walk $\psi\left[X_{n}\right]$ is a recurrent random walk on the group $\psi(\mathfrak{G})$. This group is isomorphic to the integers $Z$ since $\psi(\mathfrak{G}) / Z$ is finite. Therefore the random walk $\psi\left[X_{n}\right]$ has mean 0 , and that completes the proof of (c).

To go the other way observe that the imbedded random walk is recurrent, so that it will suffice to show that the imbedded random walk $X_{T_{n}}$ has finite variance when (c) holds. Let $X_{0}=0, X_{T_{1}}=U_{1}$, and $X_{n}=Y_{1}+Y_{2}+\ldots+Y_{n}$, where the random variables $Y_{k}$ are independent with probability measure $\mu(x)=P\left[Y_{k}=x\right]$. Then

$$
E_{0}\left[\psi\left(U_{1}\right)^{2}\right]=E_{0}\left\{\left[\psi\left(\sum_{k=1}^{T_{1}} Y_{k}\right)\right]^{2}\right\}=E_{0}\left\{\left[\sum_{k=1}^{T_{1}} \psi\left(Y_{k}\right)\right]^{2}\right\}=E_{0}\left[T_{1}\right] E\left[\psi\left(Y_{1}\right)^{2}\right],
$$

by a form of Wald's identity (cf. [5], p. 351). Now $E_{0}\left[T_{1}\right]<\infty$ because $\mathbb{G} / \mathfrak{S}$ is finite and the second moment of $\psi\left(Y_{1}\right)$ is finite by condition (c), and that completes the proof.

Remark. As remarked at the end of Section 3, the boundary theory for recurrent random walk on $\mathbb{S}$ is radically different for type III random walk. On the basis of Theorems 3.3 and 4.5 the usual Martin boundary construction (see [10], § 31) gives the following classification for the non-negative solutions $f$ of

They are

$$
\sum_{y \neq 0} P(x, y) f(y)=f(x), x \in \mathbb{S}-\{0\} .
$$

(i) positive multiples of $a(x)$ when $\mathscr{B}$ has only elements of finite order, or when (3) has an element of infinite order which gives rise to an infinite quotient group, or when $\mathscr{B S}$ has an element $y$ of infinite order such that $\mathfrak{H}=\{n y\}$ has a finite quotient group but its homomorphism $\psi_{y}$ into the rationals satisfies

$$
\sum_{x \in \Theta}\left[\psi_{y}(x)\right]^{2} P(0, x)=\infty ;
$$

(ii) positive multiples of $a(x)+c \psi(x)$, for a suitable non empty interval of values of $c$. This occurs in all remaining cases, i.e. exactly when the equivalent conditions (a), (b), (c) in Theorem 4.5 are satisfied. The homomorphism $\psi$ may be constructed from any element of infinite order. 


\section{References}

[1]. Anscombe, F. J., Large sample theory of sequential estimation. Proc. Cambridge Philos. Soc., 48 (1952), 600-607.

[2]. Choquet, G. \& Deny, J., Sur l'equation $\mu=\mu * \sigma$. C. R. Acad. Sci. Paris, 250 (1960), 799-801.

[3]. Chung, K. L. \& Erdös, P., Probability limit theorems assuming only the first moment. Mem. Amer. Math. Soc., 6 (1951).

[4]. Chusg, K. L. \& Fuchs, W. H. J., On the distribution of values of sums of random variables. Mem. Amer. Math. Soc., 6 (1951).

[5]. Dоoв, J. L., Stochastic processes. J. Wiley, N. Y., 1953.

[6]. FeLleer, W., An introduction to probability theory and its applications, Vol. I., 2nd ed., J. Wiley, N.Y., 1957.

[7]. —-, ibid., Vol. II. To appear.

[8]. Kemeny, J. G. \& Sneld, J. L., Boundary theory for recurrent Markov chains. Trans. Amer. Math. Soc., 106 (1963), 495-520.

[9]. Rudin, W., Fourier analysis on groups. Interscience, N.Y., 1962.

[10]. Spitzer, F., Principles of random walk. Van Nostrand, Princeton, 1964.

Received December 29, 1964. 